\title{
Secretogranin III may be an indicator of paraquat-induced astrocyte activation and affects the recruitment of BDNF during this process
}

\author{
XIAONI ZHAN ${ }^{1}$, FENGRUI $\mathrm{LI}^{2}$, QIAOHONG CHU ${ }^{3}$ and HAO PANG ${ }^{1}$ \\ ${ }^{1}$ Department of Forensic Genetics and Biology, School of Forensic Medicine, China Medical University, Shenyang, \\ Liaoning 110122; ${ }^{2}$ Department of Forensic Medicine, Baotou Medical College, Baotou, Inner Mongolia 014040; \\ ${ }^{3}$ Precision Medicine and Healthcare Center, Qingdao Binhai University, Qingdao, Shandong 266555, P.R. China
}

Received April 20,2018; Accepted September 28, 2018

DOI: $10.3892 / \mathrm{ijmm} .2018 .3909$

\begin{abstract}
Astrocyte activation has been described as a multi-stage defensive response, which is characterized by the morphological alteration of astrocytes and the overexpression of intermediate filament proteins. However, the functional mechanism of the secretion system in activated astroglia remains unclear. It has previously been demonstrated that secretogranin II, a member of the granin family, may be involved in the sorting and expression of inflammatory factors and excitatory neurotransmitters in paraquat $(\mathrm{PQ})$-induced astroglial activation. Secretogranin III (SCG3) has been reported to represent an important component of the regulated secretory pathway in neuroendocrine cells; however, its role as an anchor protein of dense-core vesicles in astrocytes remains to be elucidated. In the present study, a PQ-activated U118MG astrocytoma cell model established in our previous study was used to investigate the effects of SCG3. The results revealed that SCG3 was highly expressed and subsequently released from cells in response to PQ. Inhibition of SCG3 expression via transfection with small interfering RNA partially restored astrocyte morphology, but did not affect the expression of astrocytic factors. Further studies investigating the association between SCG3 and other cellular factors were conducted, in order to determine the expression levels and subcellular localization of these proteins. Neurotrophins and inflammatory factors exhibited an increase in characteristic expression patterns, paralleling the alterations in SCG3 expression. The results further demonstrated that brain-derived neurotrophic factor partially colocalized with SCG3-positive vesicles;
\end{abstract}

Correspondence to: Professor Hao Pang, Department of Forensic Genetics and Biology, School of Forensic Medicine, China Medical University, 77 Puhe Road, Shenyang North New Area, Shenyang, Liaoning 110122, P.R. China

E-mail: hpang@cmu.edu.cn

Key words: paraquat, astrocyte activation, secretogranin III, inflammatory factors, neurotrophins however, the localization of interleukin-6 was not affected. In conclusion, SCG3 may be involved in PQ-induced astrocyte activation via regulation of the expression and selective recruitment of cellular factors, thus suggesting that SCG3 may represent an indicator of astrocyte activation.

\section{Introduction}

Astrocytes represent housekeeper cells in the central nervous system (CNS), and participate in CNS development and homeostatic regulation by secreting a wide array of signaling molecules, including classical neurotransmitters, hormone peptides, neurotrophins and inflammatory factors $(1,2)$. The neuroprotective and/or harmful roles of astrocytes depend largely on the molecules they release into, and take up from, the extracellular microenvironment (3). In addition, reactive astrogliosis is a multistage defensive response that has been observed in acute injuries, such as trauma and ischemic damage, and progressive pathological conditions, including neuroinflammation and neurodegeneration in the CNS. This process is characterized by the alteration of intermediate filament proteins, such as glial fibrillary acidic protein (GFAP) and vimentin $(4,5)$. In addition, it has previously been reported that various categories of functional genes and molecules are modulated by activated astrocytes in vivo and in vitro, including inflammatory cell regulators, extracellular transmitters and neurotrophic factors (6).

The granin family, which comprises markers of the regulated secretory pathway, contains numerous acidic proteins, including chromogranin A (CgA), chromogranin B and secretogranin II-VII. The granins are located in the secretory granules of various endocrine and neuroendocrine cells, and are involved in numerous neurological diseases (7-9). Secretogranin III (SCG3) participates in the early processing of peptide hormones within the trans-Golgi network and the subsequent secretion of these peptide hormones via the regulated secretory pathway (10). By binding with $\mathrm{CgA}$ and carboxypeptidase E (CPE) at specific domains, SCG3 acts as a molecular bridge between the core aggregate and the cholesterol-rich secretory granule membrane, and it is regulated for efficient secretion and processing of 
proopiomelanocortin (POMC)-derived peptides in endocrine cells $(11,12)$. Furthermore, SCG3 has been reported to be associated with the regulated secretory pathway in numerous types of non-endocrine cells, such as mast cells and platelets $(13,14)$. Previous studies have confirmed abundant expression of SCG3 in astrocytes and neuronal populations $(15,16)$. In addition, SCG3 has been reported to be involved in neuropathology, such as in major depressive disorder, multiple sclerosis and Alzheimer's disease (AD); however, its potential role in the pathogenesis and progression of nervous system dysfunction has yet to be determined $(17,18)$.

The common pesticide paraquat (PQ), together with 6-hydroxydopamine, 1-methyl-4-phenyl-1,2,3,6-tetrahydropyridine (MPTP) and rotenone, has been extensively used to induce the pathology of Parkinson's disease in previous experiments (19-21). In our previous studies, a PQ-activated U118MG astrocytoma cell model was established, and it was observed that secretogranin II (SCG2), another member of the granin family, was partially involved in the expression and sorting of inflammatory factors and neurotransmitters in astroglial activation. The secretory pathway component SCG3 has previously been demonstrated to act as a caspase substrate in PQ-induced dopaminergic cell apoptosis, and its transport is disrupted following PQ-induced toxicity $(16,22,23)$. However, its role as an anchor protein of dense-core vesicles (DCVs) remains to be elucidated. Therefore, in the present study, the PQ-activated astroglial activation model was used to investigate the role of SCG3 in this process.

\section{Materials and methods}

Materials. Dulbecco's modified Eagle's medium (DMEM), penicillin, streptomycin and fetal bovine serum (FBS) were obtained from Gibco; Thermo Fisher Scientific, Inc. (Waltham, MA, USA). PQ dichloride and Hoechst 33258 were purchased from Sigma-Aldrich; Merck KGaA (Darmstadt, Germany). RNAiso Plus, PrimeScript RT Reagent kit and SYBR Prime EX Taq were obtained from Takara Biotechnology Co., Ltd. (Dalian, China). The Bicinchoninic Acid Protein Assay kit, SDS-PAGE gel preparing kit, bovine serum albumin (BSA), radioimmunoprecipitation assay (RIPA) lysis buffer and protease inhibitor were purchased from Beyotime Institute of Biotechnology (Shanghai, China), and the Easysee chemiluminescence reagent was obtained from TransGen Biotech, Co., Ltd. (Beijing, China). Polyvinylidene difluoride (PVDF) membranes were purchased from Merck KGaA. Antibodies against $\beta$-actin (cat. no. sc-47778) and SCG3 (cat. no. sc-50289) were obtained from Santa Cruz Biotechnology, Inc. (Dallas, TX, USA), and anti-GFAP (cat. no. WL0836) and anti-S100-calcium binding protein $\beta$ (S100ß; cat. no. WL00789) antibodies were purchased from Wanleibio Co., Ltd. (Shenyang, China). Anti-interleukin (IL)-6 (cat. no. ab9324) and anti-brain-derived neurotrophic factor (BDNF; cat. no. H00000627-M02) were purchased from Abcam (Cambridge, MA, USA) and Abnova (Taipei, Taiwan), respectively. In addition, Alexa Fluor ${ }^{\circledR}$ 594-conjugated donkey anti-rabbit immunoglobulin $\mathrm{G}(\mathrm{IgG})$ secondary antibody (cat. no. A-21207), Alexa Fluor ${ }^{\circledR}$ 488-conjugated goat anti-mouse IgG secondary antibody (cat. no. A-11029) and Lipofectamine ${ }^{\circledR} 3000$ were purchased from Invitrogen;
Thermo Fisher Scientific, Inc. SCG3 small interfering RNAs (siRNA_001-003) and the negative control (NC) siRNA (cat. no. siN05815122147) were obtained from Guangzhou RiboBio Co., Ltd. (Guangzhou, China).

Cell line. Human astrocytoma U118MG cells (cat.no. TCHu216) were obtained from Shanghai Institutes for Biological Science (Shanghai, China), and grown in DMEM High Glucose, supplemented with $10 \% \mathrm{FBS}$ and $1 \%$ penicillin-streptomycin $(10,000 \mathrm{U} / \mathrm{ml}$ and $10,000 \mu \mathrm{g} / \mathrm{ml} \mathrm{v} / \mathrm{v}$, respectively). U118MG astroglia $\left(5 \times 10^{6}\right)$ were seeded in $10 \mathrm{~cm}^{2}$ tissue culture dishes, grown at $37^{\circ} \mathrm{C}$ in a humidified incubator containing $5 \% \mathrm{CO}_{2}$, and were exposed to $0.25 \mathrm{mM}$ PQ for $48 \mathrm{~h}$, in order to establish an activated astroglia model, as described previously (23). PQ treatment was performed in accordance with ISO 15190:2003 Medical laboratories-Requirements for safety (24).

Western blotting. Cultured cells treated with $0.25 \mathrm{mM} P Q$ at $37^{\circ} \mathrm{C}$ for various durations $(0-96 \mathrm{~h})$ were homogenized in ice-cold RIPA with protease inhibitor. Subsequently, protein concentration was detected using the bicinchoninic acid method. In order to collect the conditioned media, cells were grown in serum-starved medium and were treated with $0.25 \mathrm{mM}$ PQ at $37^{\circ} \mathrm{C}$ for $0-96 \mathrm{~h}$; the medium containing PQ was replaced every $24 \mathrm{~h}$. Subsequently, the supernatant was separated after centrifugation at 2,000 $\mathrm{x} g$ for $5 \mathrm{~min}$ to remove dislodged cells. The post-nuclear lysate (30 $\mu \mathrm{g} / \mathrm{lane})$ and secretory proteins in conditioned media (30 $\mu 1 /$ lane) were separated by $10 \%$ SDS-PAGE and were transferred to PVDF membranes. The membranes were then blocked in a solution containing 5\% non-fat milk powder in Tris-buffered saline containing $0.05 \%$ Tween-20 (TBST) for $2 \mathrm{~h}$ at room temperature. Subsequently, the membranes were incubated with anti- $\beta$-actin $(1: 1,000)$ and anti-SCG3 $(1: 100)$ primary antibodies in blocking buffer overnight at $4^{\circ} \mathrm{C}$. After three washes in TBST, the membranes were incubated for a further $2 \mathrm{~h}$ at room temperature with peroxidase-conjugated goat anti-mouse antibody (1:5,000; cat. no. ZB-2305) and peroxidase-conjugated goat anti-rabbit antibody (1:2,000; cat. no. ZB-2301) (both ZSGB-BIO Technologies, Inc., Beijing, China). Bound antibodies were visualized via Easysee chemiluminescence reagent using a 5500 Luminescent imaging workstation (Tanon Science \& Technology Co., Ltd., Shanghai, China). Densitometric analyses of the bands were semi-quantified using ImageJ software V1.8.0 (National Institutes of Health, Bethesda, MD, USA), with $\beta$-actin as an internal reference.

Reverse transcription-quantitative polymerase chain reaction (RT-qPCR) analysis. Cultured cells were treated with $0.25 \mathrm{mM}$ PQ at $37^{\circ} \mathrm{C}$ for $0-72 \mathrm{~h}$. To perform RT-qPCR experiments, total RNA was isolated from cultured astroglia using RNAiso Plus, in accordance with the manufacturer's protocol. The obtained RNA was then reverse transcribed to cDNA using a PrimeScript ${ }^{\mathrm{TM}}$ RT Reagent kit in a $20-\mu 1$ reaction mixture containing $4 \mu \mathrm{l}$ 5X PrimeScript buffer, $1 \mu \mathrm{l}$ PrimeScript RT Enzyme Mix I, $1 \mu 1$ Oligo dT Primer, $1 \mu$ l Random 6 primers and $1 \mu \mathrm{g}$ total RNA, under the following thermocycling conditions: $37^{\circ} \mathrm{C}$ for $15 \mathrm{~min}$ followed by $85^{\circ} \mathrm{C}$ for $5 \mathrm{sec}$, in accordance with the manufacturer's protocol. Subsequently, cDNA was amplified using SYBR Prime EX Taq via an ABI 7500 
Real-Time PCR system (Applied Biosystems; Thermo Fisher Scientific, Inc.) with the following primers: SCG3 forward, 5'-TGTTAGTGCTCCCGATTCAAG-3' and reverse, 5'-TCA AAGGTCTTTCTGCACTTAATTC-3'; and $\beta$-actin forward, 5'-AGATGACCCAGATCATGTTTG-3' and reverse, 5'-ATC ACGATGCCAGTGGTA-3'. The thermocycling conditions used were as follows: Initial denaturation at $95^{\circ} \mathrm{C}$ for $30 \mathrm{sec}$, followed by 40 cycles at $95^{\circ} \mathrm{C}$ for $5 \mathrm{sec}$ and $60^{\circ} \mathrm{C}$ for $34 \mathrm{sec}$. Each sample was tested in triplicate wells and the experiment was repeated at least three times. $\beta$-actin was used as an internal reference, and a positive PCR control was performed. The results were quantified using in the $2^{-\Delta \Delta C q}$ method (25).

Transfection with siRNA. U118MG cells (cell density 70\%) were transfected with SCG3 siRNA or NC siRNA $(\sim 100 \mathrm{nM})$ using Lipofectamine ${ }^{\circledR} 3000$ and were treated with PQ immediately after transfection; RT-qPCR was conducted 48 h later to confirm successful knockdown of SCG3 mRNA in vitro. A total of $48 \mathrm{~h}$ post-transfection, total RNA was isolated, and the mRNA expression levels of SCG3 and other astrocytic factors were determined using RT-qPCR. The primers used for the amplification of astrocytic factors were described in a previous study (23). The sequences of the most efficient SCG3 siRNA (siRNA_002) were: Sense, 5'-GAACAATATCTCCAGAAG AdTdT-3', and antisense, 5'-UCUUCUGGAGAUAUUGUU CdTdT-3'. In addition, cell morphology was observed under phase contrast microscopy (Leica Microsystems, Inc., Buffalo Grove, IL, USA) post-transfection with SCG3 siRNA.

Fluorescence microscopy. Cultured cells were grown on sterile glass cover slips at $37^{\circ} \mathrm{C}$ overnight. After incubation under experimental conditions (control or $0.25 \mathrm{mM}$ PQ-treated for $48 \mathrm{~h}$ ), cells were washed twice with PBS containing $1 \mathrm{mM}$ $\mathrm{MgCl}_{2}$ and $1 \mathrm{mM} \mathrm{CaCl}_{2}(\mathrm{CM})$, and were fixed with $4 \%$ paraformaldehyde solution for $15 \mathrm{~min}$. For permeabilization, the fixed cells were washed in PBS/CM and were incubated for $10 \mathrm{~min}$ at room temperature with $0.2 \%$ Triton $\mathrm{X}-100$ in $\mathrm{PBS} / \mathrm{CM}$. After three washes with PBS/CM, the cells were blocked with $\mathrm{PBS} / \mathrm{CM}$ containing $0.2 \% \mathrm{BSA}$ for $30 \mathrm{~min}$ at room temperature. The cells were subsequently incubated with anti-S100 $\beta$, anti-GFAP, anti-SCG3 and/or anti-IL-6 and anti-BDNF antibodies (1:50 in PBS/CM containing 0.2\% BSA) overnight at $4^{\circ} \mathrm{C}$. After three further washes with $\mathrm{PBS} / \mathrm{CM}$, the cells were incubated with Alexa Fluor ${ }^{\circledR}$ 488-conjugated secondary antibody (1:2,000 in PBS/CM) and/or Alexa Fluor ${ }^{\circledR}$ 594-conjugated secondary antibody (1:2,000 in PBS/CM) for $1 \mathrm{~h}$ at room temperature. Finally, the cells were washed three times with PBS/CM and incubated with Hoechst 33258, in accordance with the manufacturer's protocol. The cells were observed under a fluorescence microscope or confocal microscope (Leica Microsystems, Inc.). Semi-quantitative data were obtained from at least five randomly selected visual fields from the three different assays. Identical acquisition parameters were applied when cells were labeled with the same antibody. The process by which the number of cells were counted was double-blind and the labeled cell body areas were measured using ImageJ software V1.8.0 (National Institutes of Health), according to the methods described in a previous study (26). Colocalization was measured using the Co-Localization function of Image Pro Plus 6.0 software (Media Cybernetics, Inc., Rockville,
MD, USA). Finally, the density of each image was normalized against the mean value of the controls and presented as arbitrary units of the control. Pearson's correlation coefficient and the overlay percentage of SCG3 expression from five images per condition were used to determine colocalization.

Statistical analysis. For statistical analysis, all quantitative data were collected from at least three independent experiments and the final data were expressed as the means \pm standard deviation. Statistically significant differences between three or more groups were determined via one-way analysis of variance followed by Dunnett's post hoc test using GraphPad Prism 6 (GraphPad Software, Inc., La Jolla, CA, USA). The differences in immunofluorescence between the PQ-treated group and the control group were determined using the Student's t-test. $\mathrm{P}<0.05$ was considered to indicate a statistically significant difference.

\section{Results}

mRNA and protein expression levels of SCG3 are upregulated in $P Q$-activated U118MG astroglia. In our previous study, a PQ-activated U118MG astroglia model was established in vitro; the results revealed that the cell cycle and expression levels of astrocytic factors are markedly altered following treatment with PQ (23). To further investigate the activity of U118MG astroglia, the expression levels of GFAP and S100 $\beta$, which are commonly used as markers of astroglial activation, were determined in the present study. As presented in Fig. 1, the results of a cell immunofluorescence analysis revealed that GFAP and S100 $\beta$ proteins were overexpressed in U118MG astroglia following $48 \mathrm{~h}$ of PQ treatment, thus suggesting that the cells were in a state of activation (Fig. 1A and B).

The expression levels of the regulated secretory pathway component SCG3 were subsequently investigated. The results of RT-qPCR revealed that the mRNA expression levels of SCG3 were significantly increased (2.1-fold) at $48 \mathrm{~h}$, and were slightly decreased at $72 \mathrm{~h}$ compared with at $48 \mathrm{~h}$ (Fig. 2A). Furthermore, the protein expression levels of SCG3 were significantly increased at the $48 \mathrm{~h}$ time point and continued to gradually increase in a time-dependent manner, which was mostly consistent with the mRNA expression results (Fig. 2B and C).

Subcellular localization and secretion of SCG3 are altered in PQ-activated U118MG astroglia. In the present study, the effects of PQ on the morphology of U118MG cells were investigated; the results demonstrated that the mean number of cell processes and area of cell bodies were markedly increased following treatment with PQ for $48 \mathrm{~h}$, thus suggesting that the astroglia developed a hypertrophic and bushy phenotype following activation (Fig. 3A). Subsequently, the expression and distribution of SCG3 at the subcellular level were determined via immunofluorescence. The results revealed that SCG3 was abundantly expressed and distributed evenly throughout the cytoplasm and processes of U118MG cells. However, when astroglia were activated by $\mathrm{PQ}$, the relative fluorescence intensity of SCG3 was markedly increased. Morphologically, punctate-patterned SCG3 markedly accumulated in the perinuclear region in hypertrophic U118MG 
A

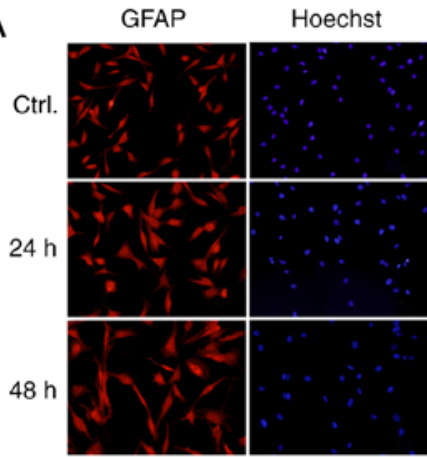

$\mathrm{S} 100 \beta$

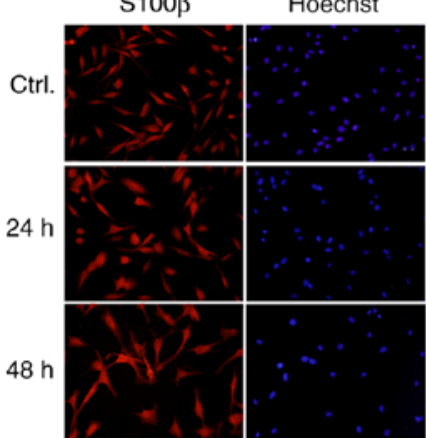

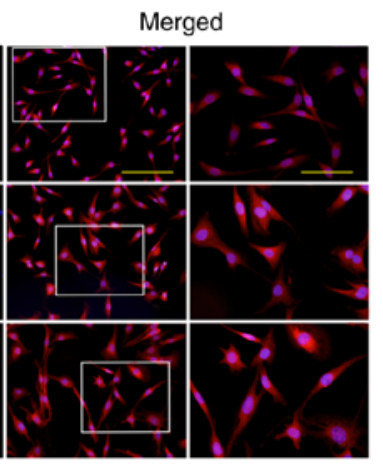

Merged

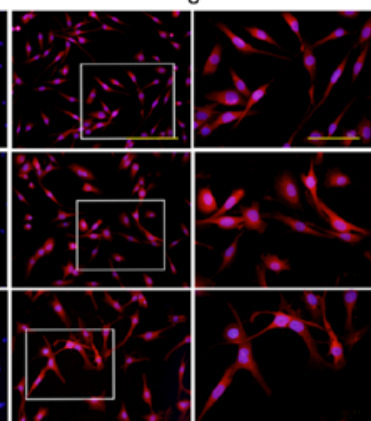

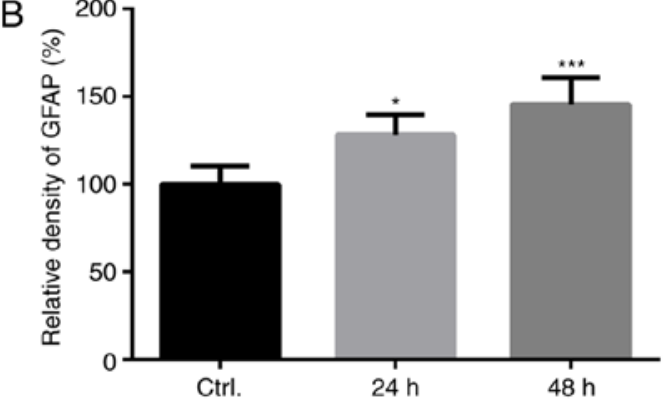

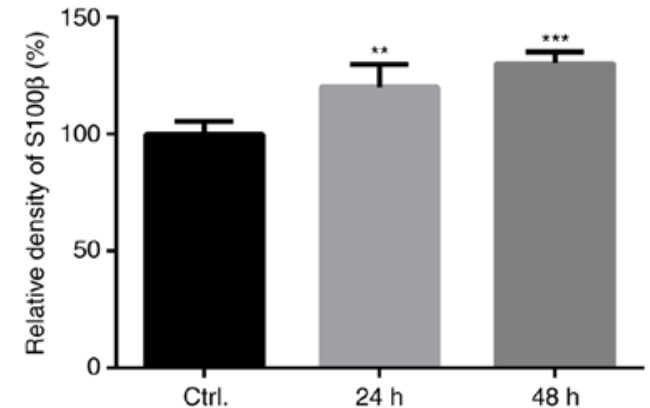

Figure 1. Reactivity of PQ-activated astroglia. (A) Astrocytic cytoskeletal markers, GFAP and S100 $\beta$, were immunostained in U118MG astroglia (red) before and after PQ treatment. Nuclei were counterstained blue with Hoechst 33258. Scale bar, $200 \mu \mathrm{m}$. (B) Semi-quantification of the relative expression levels of GFAP and S100 $\beta$ was performed using ImageJ software. Data are presented as the means \pm standard deviation $(\mathrm{n}=5)$. ${ }^{*} \mathrm{P}<0.05,{ }^{* *} \mathrm{P}<0.01$ and ${ }^{* * *} \mathrm{P}<0.001 . \mathrm{GFAP}$, glial fibrillary acidic protein; PQ, paraquat; $100 \beta$, calcium-binding protein $\beta$.
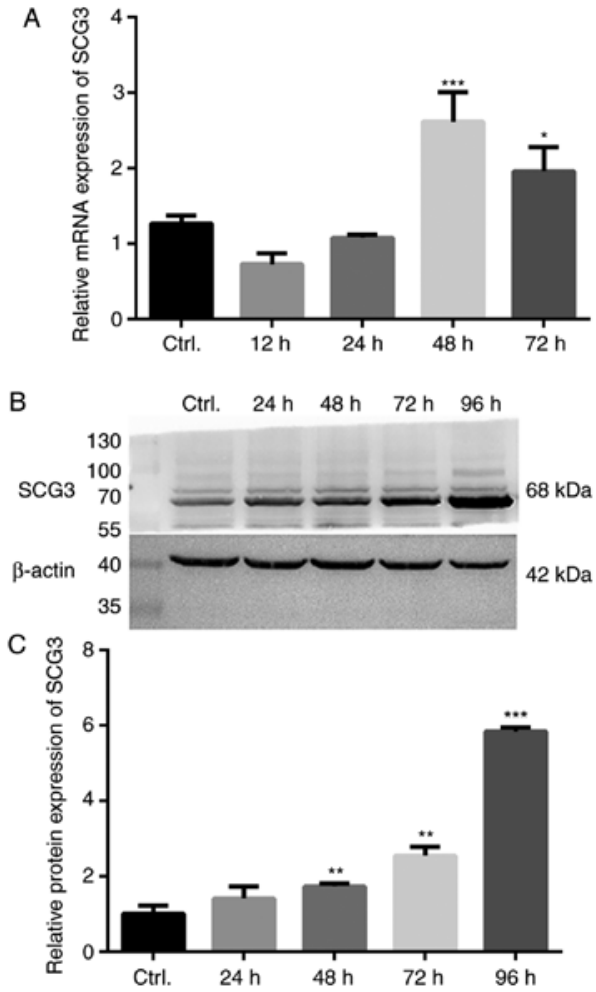

Figure 2. mRNA and protein expression levels of SCG3 in PQ-activated astroglia. (A) SCG3 mRNA expression was analyzed via reverse transcription-quantitative polymerase chain reaction following treatment with $\mathrm{PQ} ; \beta$-actin was used as an internal reference gene. (B) SCG3 protein expression in U118MG astroglia following PQ treatment was analyzed by immunoblotting ( $30 \mu \mathrm{g}$ total protein loaded per well). (C) Protein expression levels of SCG3 were semi-quantified by densitometric analysis and were normalized to $\beta$-actin. Data are presented as the means \pm standard deviation $(\mathrm{n}=3)$. ${ }^{*} \mathrm{P}<0.05,{ }^{* *} \mathrm{P}<0.01$ and ${ }^{* * * *} \mathrm{P}<0.001$ compared with the control group. PQ, paraquat; $\mathrm{SCG} 3$, secretogranin III. astroglia (Fig. 3B and C), thus indicating that a large number of SCG3-positive vesicles was rapidly synthesized in the cells following treatment with PQ. In addition, to determine the levels of SCG3 protein secreted from PQ-activated astroglia, conditioned culture media were collected at intervals of $24 \mathrm{~h}$ and were further analyzed. When equal volumes of media were loaded, it was revealed that levels of externally secreted SCG3 were increased in activated astroglia between 24 and $72 \mathrm{~h}$ (Fig. 3D and E).

SCG3 knockdown partially attenuates $P Q$-induced alterations to astrocytic morphology, but does not affect the expression levels of astrocytic factors. To further confirm the involvement of SCG3 in PQ-stimulated U118MG astroglial activation, the effects of PQ on SCG3 and astrocytic factor expression were determined after knockdown of SCG3 via siRNA transfection. Firstly, the efficiency of three candidate SCG3 siRNAs (siRNA_001-003) was investigated, and the siRNA that exerted the most marked effect (siRNA_002) was selected for use in subsequent experiments (Fig. 4A). The results, as observed by phase contrast microscopy revealed that swelling of U118MG cells was partially attenuated by transfection with SCG3 siRNA (Fig. 4B). The RT-qPCR results revealed that SCG3 expression was markedly decreased (0.22-fold) following transfection with SCG3 siRNA compared with the $\mathrm{NC}$ group $(\mathrm{P}<0.05)$. In subsequent experiments, U118MG cells were treated with $0.25 \mathrm{mM}$ PQ immediately after SCG3 siRNA transfection; RT-qPCR was conducted after $48 \mathrm{~h}$. The results demonstrated that $\mathrm{SCG} 3$ expression was significantly decreased in the siRNA_002 + PQ group compared with in the PQ group $(\mathrm{P}<0.05$; Fig. $4 \mathrm{C})$. Furthermore, the expression 
A

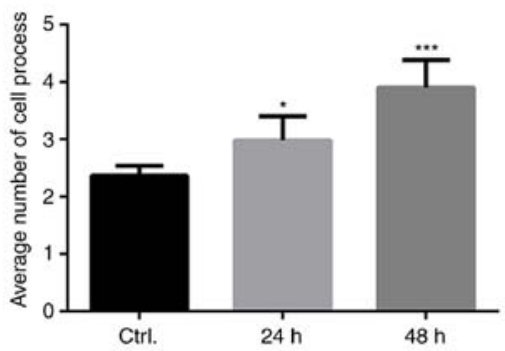

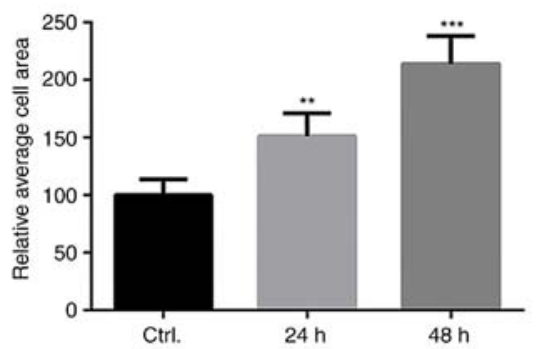
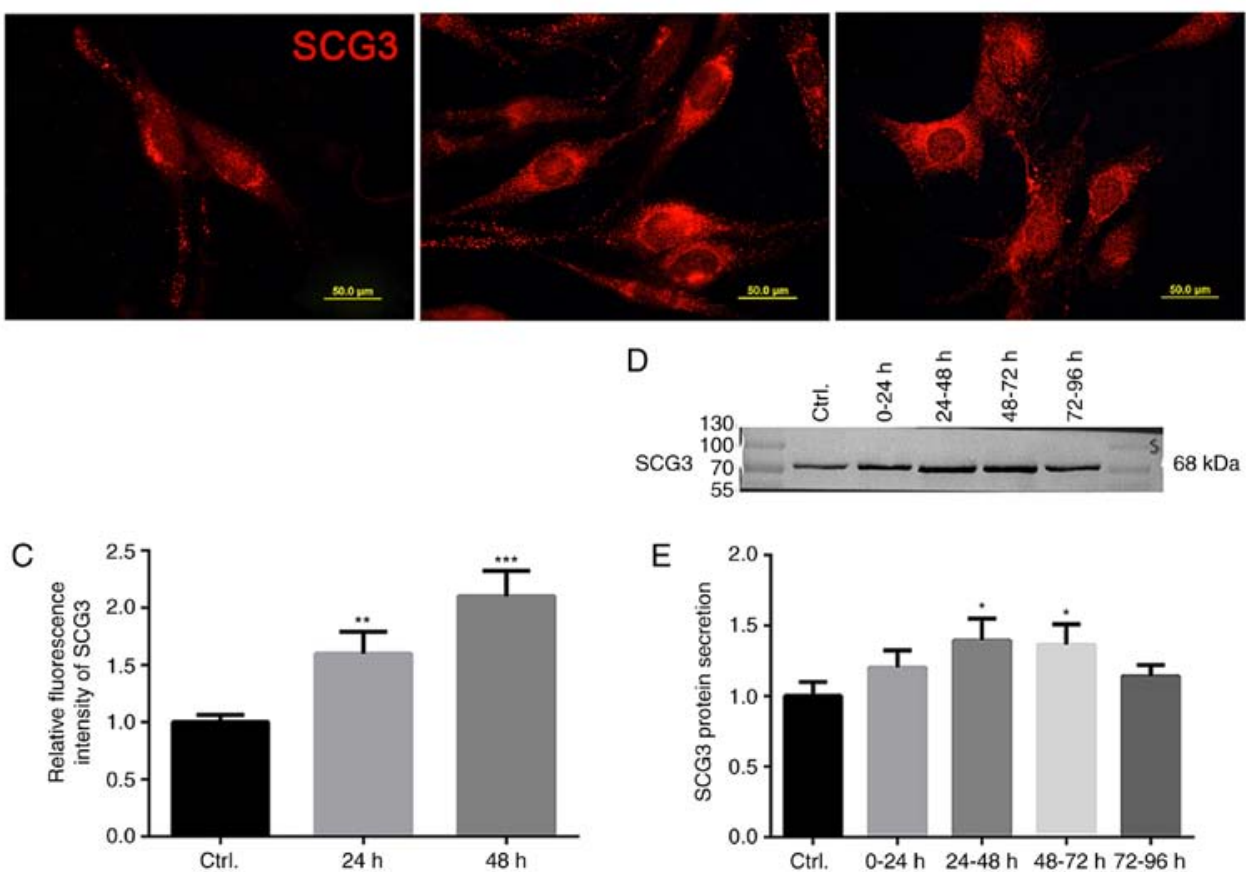

$\mathrm{E}$

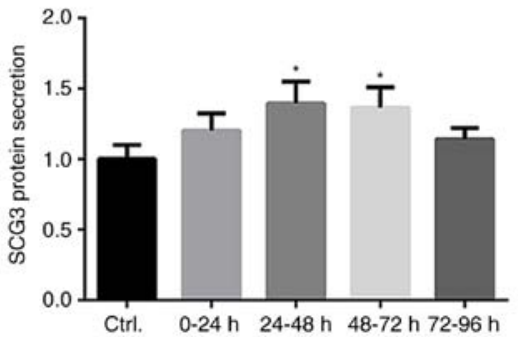

Figure 3. Subcellular localization and secretion of SCG3 in PQ-activated astroglia. (A) Mean number of cell processes and cell body area were determined using a double-blind method and ImageJ software $(\mathrm{n}=5$ ). (B) U118MG cells were fixed and immunostained to determine subcellular localization of SCG3 (red). (C) Semi-quantification of the relative fluorescence intensities of SCG3 was performed using ImageJ software (n=5). (D) Basal and PQ-stimulated release of SCG3 from U118MG cells at $24 \mathrm{~h}$ intervals as analyzed by immunoblotting (30 $\mu 1$ conditioned medium was loaded). (E) SCG3 secretion was semi-quantified by densitometric analysis $(\mathrm{n}=3)$. Data are presented as the means \pm standard deviation. ${ }^{*} \mathrm{P}<0.05,{ }^{* *} \mathrm{P}<0.01$ and ${ }^{* * *} \mathrm{P}<0.001$ compared with the control group. PQ, paraquat; SCG3, secretogranin III.

levels of various astrocyte-derived factors were investigated following SCG3 knockdown in the present study, including GFAP, S100 $\beta$, glutamine synthetase (GS) and glutamate transporter (GLT-1) astrocyte-specific proteins; BDNF and glial-derived neurotrophic factor (GDNF) neurotrophic factors; and tumor necrosis factor (TNF- $\alpha$ ), IL-1 $\beta$ and IL-6 inflammatory cytokines (Fig. 4D). However, no significant alterations in the mRNA expression levels of these factors were detected between the SCG3 knockdown group and the NC group.

Intracellular SCG3-positive DCVs may participate in the trafficking of BDNF and IL-6 in PQ-activated astroglia. It has previously been reported that there are numerous DCV variants present in astrocytes (27); however, whether SCG3-positive DCVs are present in astroglia remains unknown. Despite the present results revealing that there were no marked associations between SCG3 and gliotransmitters (data not shown), other potential associations between neurotrophic BDNF and neuropoietic cytokine IL-6 with SCG3-positive DCVs were investigated via colocalization analysis in PQ-treated U118MG astroglia. In PQ-activated astroglia, co-distribution of BDNF immunoreactivity with SCG3-positive vesicles was observed, and a vesicular pattern of both BDNF and SCG3 staining was visualized in the perinuclear space and cytoplasm. However, overlap of IL-6 and SCG3 expression was only observed in the perinuclear region, which may be due to early synthesis of the corresponding proteins in the endoplasmic reticulum (ER; Fig. 5A). In Pearson's correlation coefficient analysis, the overlapping was determined by colocalization index: $0.0-0.2$ is considered to indicate an extremely weak or no correlation, whereas 0.2-0.4 and 0.4-0.6 are considered weak and moderate correlations, respectively. In the control groups, slight colocalization was demonstrated between BDNF and SCG3, IL-6 and SCG3 (0.23 and 0.1, respectively). However, the colocalizations were increased following PQ treatment $(0.43$ and 0.34 , respectively). Furthermore, when the percentage of overlay in the cytoplasm and processes was measured, the overlay of SCG3 with BDNF following PQ-induced activation was increased from 13 to $20.5 \%$ ( $\mathrm{P}<0.05)$, whereas the overlay of SCG3 with IL-6 was increased from 7.4 to $8.8 \%$ (Fig. 5B). These results indicated that SCG3 DCVs possibly affect the recruitment of BDNF, but not IL-6, in U118MG cells during PQ-induced astrocyte activation. 

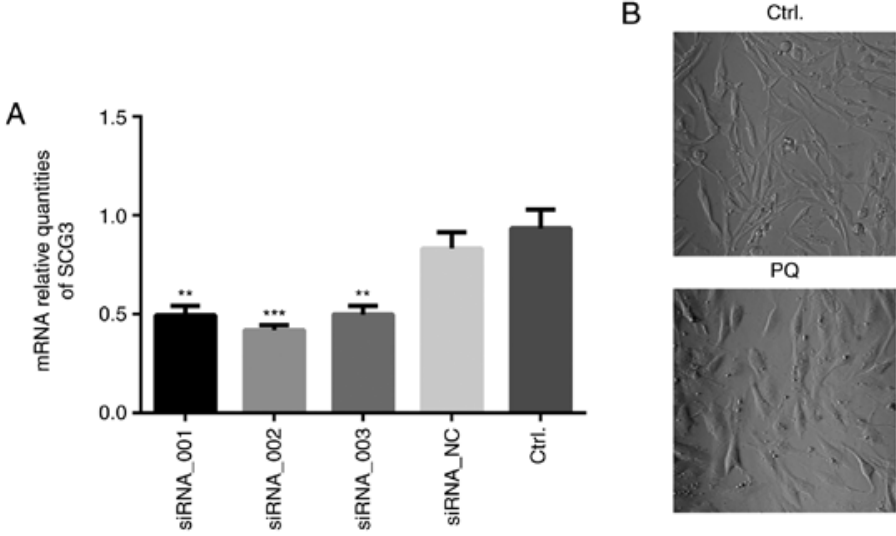

$\mathrm{PQ}$
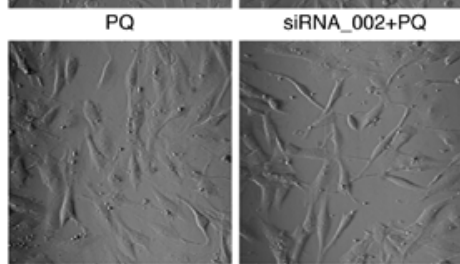

Ctrl.

NC
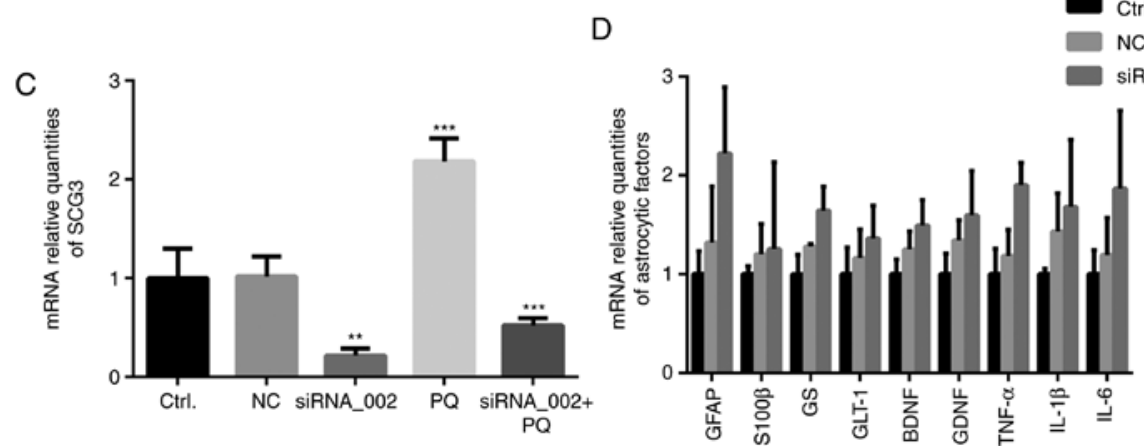

Figure 4. Cell morphology and mRNA expression levels of astrocytic factors in U118MG astroglia following knockdown of SCG3. (A) SCG3 mRNA expression levels were analyzed by RT-qPCR post-transfection with three candidate siRNAs $(n=3)$. SCG3 expression levels were presented relative to $\beta$-actin (B) Astroglial swelling was partially attenuated by SCG3 siRNA under phase contrast microscopy (magnification, x20). (C) RT-qPCR was used to detect SCG3 expression levels following SCG3 knockdown. (D) mRNA expression levels of astrocyte-specific functional proteins in U118MG cells were analyzed by RT-qPCR post-transfection with siRNA_002 $(\mathrm{n}=3)$. Data are presented as the means \pm standard deviation. ${ }^{* *} \mathrm{P}<0.01$ and ${ }^{* * *} \mathrm{P}<0.001$ compared with the control group. BDNF, brain-derived neurotrophic factor; GDNF, glial-derived neurotrophic factor; GFAP, glial fibrillary acidic protein; GLT-1, glutamate transporter-1; GS, glutamine synthetase; IL, interleukin; NC, non-specific control; PQ, paraquat; RT-qPCR, reverse transcription-quantitative polymerase chain reaction; S100 $\beta$, S100-calcium-binding protein $\beta$; SCG3, secretogranin III; siRNA, small interfering RNA; TNF- $\alpha$, tumor necrosis factor- $\alpha$.

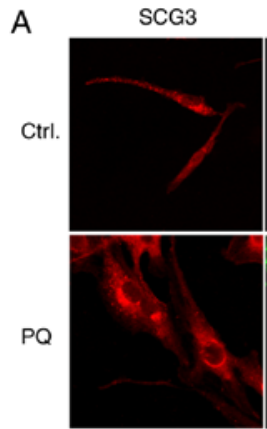

SCG3

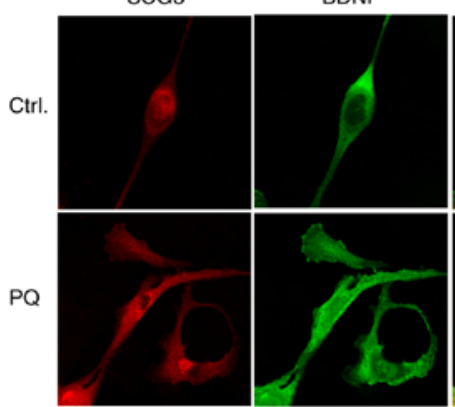

IL-6
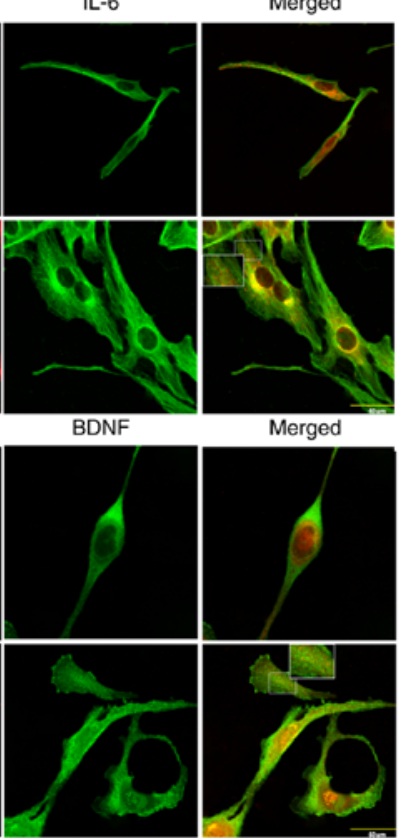
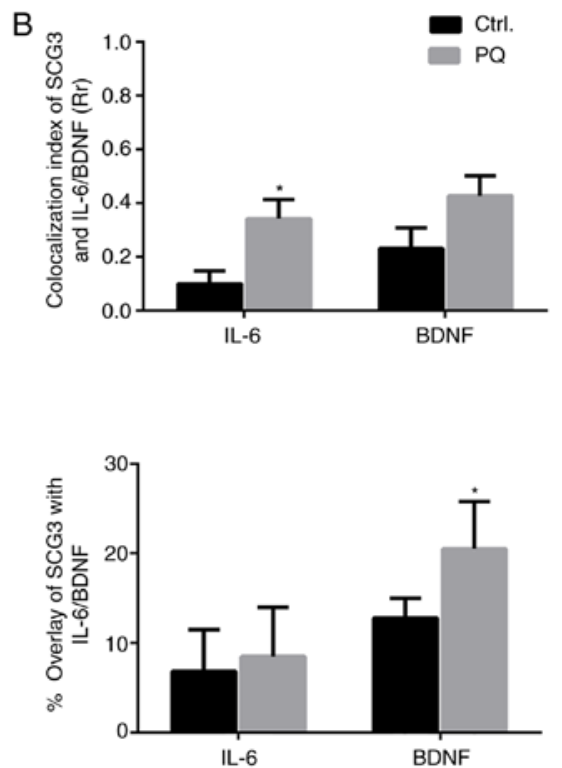

Figure 5. Co-staining of IL-6 and BDNF with SCG3-positive DCVs. (A) U118MG cells were fixed and immunostained following PQ treatment, in order to determine subcellular distribution of SCG3-positive DCVs (red) and BDNF (green). Overlap of SCG3 and BDNF expression was observed in the perinuclear space and cytoplasm of activated astroglia. Subcellular distribution of SCG3-positive DCVs (red) and IL-6 (green) was also determined. The majority of SCG3 and IL-6 overlapped expression was accumulated in the perinuclear space of activated astroglia following treatment with PQ. (B) Quantification of colocalization was determined by Pearson's correlation coefficient, and the percentage overlay of SCG3 with IL-6 and BDNF from five images was determined. Data are presented as the means \pm standard deviation $(\mathrm{n}=5)$. " $\mathrm{P}<0.05$ compared with the control group. BDNF, brain-derived neurotrophic factor; IL, interleukin; PQ, paraquat; SCG3, secretogranin III; Rr, colocalization index of Pearson's correlation coefficient. 


\section{Discussion}

At present, GFAP is the most commonly used hallmark for astrocyte activation; however, GFAP overexpression is not always observed during the activation of all astrocytes. For example, astrocytes activated by ATP, IL-1 and MPTP may express unmodified and even decreased levels of GFAP (28-30). In addition, the role of GFAP in neuronal regeneration, scar formation and amyloid load assimilation remains unclear $(31,32)$. Therefore, the present study aimed to identify an indicator that reflects functional modifications occurring during the activation of astrocytes. Previous studies have demonstrated that numerous transporters and carrier proteins, such as vesicular transporters of $\gamma$-aminobutyric acid and glutamate (excitatory amino acid transporter 1 and 2), are affected following the activation of astrocytes $(33,34)$. In addition, two individual microarray analyses identified enhanced expression of the secretogranin family members secretogranin V and secretogranin VII in reactive astrocytes isolated from various neuropathologies $(6,20)$. In the present study, a PQ-activated astrocyte model established in our previous study was used, and the results revealed that PQ exposure led to morphological alterations, and increased expression levels of GFAP and S100 $\beta$ astrocyte activation markers in U118MG astroglia (23). In addition, a regulated secretory pathway component, SCG3, was further investigated in PQ-activated astrocytes. Our previous study revealed that PQ exposure induced the apoptosis of dopaminergic SH-SY5Y cells and decreased SCG3 expression (16). These findings indicated that alterations in SCG3 expression may vary in different cell types. In the present study, it was demonstrated that the expression levels of SCG3 were gradually increased, in accordance with the degree of U118MG astroglial activation induced by PQ treatment, thus suggesting that altered SCG3 may reflect astrocyte reactivity to a certain extent. This effect has previously been reported in brain perforation and $A D$ plaque-activated astrocytes $(15,35)$. Conversely, depletion of SCG3 via RNA interference attenuated the hypertrophic phenotype of U118MG astroglia and reversed PQ-induced increases in SCG3 expression, which further suggested that SCG3 may have an active role in PQ-induced astrocyte activation. Therefore, it was hypothesized that SCG3 secretion in astrocytes may represent an indicator of cellular activation and provide further information regarding transmitter signaling.

SCG3-positive vesicles have been identified in primary astrocyte cultures and human brain samples (35). Furthermore, aberrant accumulation of SCG3 and CPE in senile plaque-surrounding astrocytes and dystrophic neurites obtained from patients with AD suggests that SCG3 may affect neuropeptide trafficking and processing $(15,35)$. However, the mechanism by which neurotransmitters and peptides are transported by SCG3 secretory vesicles in astrocytes remains largely unknown. In the present study, both extracellular and intracellular SCG3 expression levels were upregulated in U118MG astroglia following treatment with PQ. Immunofluorescence analysis demonstrated that SCG3-positive vesicles accumulated in the perinuclear ER, where they were generated following exposure to PQ. These results indicated that PQ stimulated the biosynthesis of SCG3 secretory vesicles, thus suggesting that SCG3 may be involved in the recruitment of cellular factors during PQ-induced astrocyte activation. A previous study indicated that DCVs isolated from astrocytes contain a range of gliotransmitters, such as atrial natriuretic peptide, neuropeptide Y and ATP, which serve important roles in astrocyte metabolism (36). In our previous study, it was demonstrated that three categories of astrocytic proteins were upregulated to different extents in PQ-activated U118MG astroglia, including astrocytic marker proteins (GFAP, S100 $\beta$, GS and GLT-1), neurotrophins (BDNF and GDNF), and cytokines (TNF- $\alpha$, IL-1 $\beta$, IL-6) (23). However, the mRNA expression levels of the aforementioned proteins did not exhibit any significant alterations following the silencing of SCG3. Therefore, it was hypothesized that SCG3 secretory vesicles may be associated with the regulation of secretory peptide secretion, rather than the regulation of gene expression, in PQ-activated astrocytes.

Previous studies have demonstrated that BDNF targets to SCG2-positive DCVs in neuronal dendrites, and BDNF and SCG2 genes are co-activated in response to intracellular $\mathrm{Ca}^{2+}$ signaling $(33,37,38)$. In addition, the sorting signal of BDNF can specifically bind to the sorting receptor $\mathrm{CPE}$, which is localized closely with SCG3 at the periphery region of endocrine cells $(12,39,40)$. In the present study, it was observed that double-labeled vesicles were distributed in the perinuclear ER and cytoplasm of U118MG astroglia, and their numbers were increased during PQ stimulation. Therefore, it was hypothesized that SCG3 may act as an analogous sorting receptor to CPE during the processing and trafficking of BDNF. In addition, SCG3 is involved in inflammatory mediator biogenesis and release in mast cells, which is partially analogous to astrocytes during immune regulation (14). It has also been reported that IL-6 is stored in SCG2-positive DCVs and is released via the regulated secretory pathway following ATP stimulation in PC12 cells (41). Therefore, the role of SCG3 in IL-6 trafficking was analyzed. The results of the present study revealed that the overlap of IL- 6 and SCG3 vesicles was predominantly observed in the perinuclear space of PQ-activated U118MG astroglia, which may be due to their common biosynthesis in the ER. However, when the percentage of overlay in the cytoplasm and processes was measured, there was no significant increase in the overlay between SCG3 and IL-6 following PQ-induced activation. Therefore, it may be hypothesized that SCG3-positive vesicles exert a significant effect on BDNF trafficking but do not participate in the subsequent processing and trafficking of IL-6 in astrocytes.

In conclusion, the results of the present study revealed that the regulated secretory pathway component SCG3 was expressed in U118MG astroglia, and SCG3 expression and astrocyte activation were both gradually increased in response to PQ stimulation. Despite the fact that SCG3 knockdown did not affect the expression of other astrocytic factors, de novo SCG3 vesicles contributed to the trafficking of BDNF, but not IL-6, in PQ-activated astrocytes. These results suggested that SCG3 may act as an indicator of astrocyte activation. In addition, SCG3 may have a role in astrocyte self-sustenance as well as neuroglia peptide communication; however, the present data were derived from an in vitro astrocytoma cell line model, and only one PQ activator was used. Therefore, some limitations may exist with regards to the alteration of 
SCG3 in response to astrocyte activation. For these reasons, future experiments should be performed using primary astrocytes or animal models, and the role of SCG3 in astrocyte activation should be reported in response to other activators reported in previous studies; for example, ATP, IL-1 and MPTP (28-30). Furthermore, considering the small number of DCVs in astrocytes, further experiments to pre-concentrate and separate SCG3-positive vesicles would be beneficial to confirm the target aggregates of SCG3 and to determine its role in the pathogenesis of neurodegenerative disorders.

\section{Acknowledgements}

Not applicable.

\section{Funding}

The present study was supported by grants from the National Natural Science Foundation of China (grant nos. 81172713 and 81471826).

\section{Availability of data and materials}

All data generated or analyzed during this study are included in this published article.

\section{Authors' contributions}

XZ wrote the manuscript. XZ and QC conducted the experiments. XZ and FL analyzed the results and modified the manuscript. HP made substantial contributions to conception and design, he also revised the manuscript critically for important intellectual content. All authors read and approved the final manuscript.

\section{Ethics approval and consent to participate}

Not applicable.

\section{Patient consent for publication}

Not applicable.

\section{Competing interests}

The authors declare that they have no competing interests.

\section{References}

1. Parpura V, Heneka MT, Montana V, Oliet SH, Schousboe A, Haydon PG, Stout RF Jr, Spray DC, Reichenbach A, Pannicke T, et al: Glial cells in (patho)physiology. J Neurochem 121: 4-27, 2012.

2. Schousboe A and Waagepetersen HS: Role of astrocytes in glutamate homeostasis: Implications for excitotoxicity. Neurotox Res 8: 221-225, 2005.

3. Yu B, Changsheng Y,WenjunZ,Ben L,HaiQ,Jing M, Guangwei X, Shuhua W, Fang L, Aschner M and Rongzhu L: Differential protection of pre-versus post-treatment with curcumin, Trolox, and $\mathrm{N}$-acetylcysteine against acrylonitrile-induced cytotoxicity in primary rat astrocytes. Neurotoxicology 51: 58-66, 2015.

4. Ben Haim L, Carrillo-de Sauvage MA, Ceyzeriat K and Escartin C: Elusive roles for reactive astrocytes in neurodegenerative diseases. Front Cell Neurosci 9: 278, 2015.
5. Eng LF, Ghirnikar RS and Lee YL: Glial fibrillary acidic protein: GFAP-thirty-one years (1969-2000). Neurochem Res 25: 1439-1451, 2000.

6. Zamanian JL, Xu L, Foo LC, Nouri N, Zhou L, Giffard RG and Barres BA: Genomic analysis of reactive astrogliosis. J Neurosci 32: 6391-6410, 2012.

7. Taupenot L, Harper KL and O'Connor DT: The chromogranin-secretogranin family. N Engl J Med 348: 1134-1149, 2003.

8. Bartolomucci A, Possenti R, Mahata SK, Fischer-Colbrie R, Loh YP and Salton SR: The extended granin family: Structure, function, and biomedical implications. Endocr Rev 32: 755-797, 2011.

9. van Luijn MM, van Meurs M, Stoop MP, Verbraak E, Wierenga-Wolf AF, Melief MJ, Kreft KL, Verdijk RM, 't Hart BA, Luider TM, et al: Elevated expression of the cerebrospinal fluid disease markers chromogranin a and clusterin in astrocytes of multiple sclerosis white matter lesions. J Neuropathol Exp Neurol 75: 86-98, 2016.

10. Hosaka M and Watanabe T: Secretogranin III: A bridge between core hormone aggregates and the secretory granule membrane. Endocr J 57: 275-286, 2010.

11. Hosaka M, Suda M, Sakai Y, Izumi T, Watanabe T and Takeuchi T: Secretogranin III binds to cholesterol in the secretory granule membrane as an adapter for chromogranin A. J Biol Chem 279: 3627-3634, 2004.

12. Hosaka M, Watanabe T, Sakai Y, Kato T and Takeuchi T: Interaction between secretogranin III and carboxypeptidase $\mathrm{E}$ facilitates prohormone sorting within secretory granules. J Cell Sci 118: 4785-4795, 2005.

13. Coppinger JA, Cagney G, Toomey S, Kislinger T, Belton O, McRedmond JP, Cahill DJ, Emili A, Fitzgerald DJ and Maguire PB: Characterization of the proteins released from activated platelets leads to localization of novel platelet proteins in human atherosclerotic lesions. Blood 103: 2096-2104, 2004.

14. Prasad P, Yanagihara AA, Small-Howard AL, Turner H and Stokes AJ: Secretogranin III directs secretory vesicle biogenesis in mast cells in a manner dependent upon interaction with chromogranin A. J Immunol 181: 5024-5034, 2008.

15. Paco S, Pozas E and Aguado F: Secretogranin III is an astrocyte granin that is overexpressed in reactive glia. Cereb Cortex 20: 1386-1397, 2010.

16. Li F, Tian X, Zhou Y, Zhu L, Wang B, Ding M and Pang H: Dysregulated expression of secretogranin III is involved in neurotoxin-induced dopaminergic neuron apoptosis. J Neurosci Res 90: 2237-2246, 2012.

17. Teunissen CE, Koel-Simmelink MJ, Pham TV, Knol JC, Khalil M, Trentini A, Killestein J, Nielsen J, Vrenken H, Popescu V, et al: Identification of biomarkers for diagnosis and progression of MS by MALDI-TOF mass spectrometry. Mult Scler 17: 838-850, 2011

18. Teyssier JR, Ragot S, Chauvet-Gélinier JC, Trojak B and Bonin B: Activation of a $\triangle F O S B$ dependent gene expression pattern in the dorsolateral prefrontal cortex of patients with major depressive disorder. J Affect Disord 133: 174-178, 2011.

19. Berry C, La Vecchia C and Nicotera P: Paraquat and Parkinson's disease. Cell Death Differ 17: 1115-1125, 2010.

20. Nakagawa T and Schwartz JP: Gene expression profiles of reactive astrocytes in dopamine-depleted striatum. Brain Pathol 14: 275-280, 2004

21. Sandström J, Broyer A, Zoia D, Schilt C, Greggio C, Fournier M, Do KQ and Monnet-Tschudi F: Potential mechanisms of development-dependent adverse effects of the herbicide paraquat in 3D rat brain cell cultures. Neurotoxicology 60: 116-124, 2017.

22. Li F, Tian $X$, Zhan $X$, Wang $B$, Ding $M$ and Pang $H$ : Clathrin-dependent uptake of paraquat into SH-SY5Y cells and its internalization into different subcellular compartments. Neurotox Res 32: 204-217, 2017.

23. Zhan X, Li F, Chu Q and Pang H: Effects of PQ's cytotoxicity on secretory vesicles in astroglia: Expression alternation of secretogranin II and its potential interaction with intracellular factors. Biochem Biophys Res Commun 497: 675-682, 2018.

24. Noble MA: ISO 15190:2003 medical laboratories-requirements for safety. EJIFCC 15: 141-143, 2004.

25. Livak KJ and Schmittgen TD: Analysis of relative gene expression data using real-time quantitative PCR and the 2(-Delta Delta C(T)) method. Methods 25: 402-408, 2001.

26. Sazonova OV, Lee KL, Isenberg BC, Rich CB, Nugent MA and Wong JY: Cell-cell interactions mediate the response of vascular smooth muscle cells to substrate stiffness. Biophys J 101: 622-630, 2011. 
27. Verkhratsky A, Matteoli M, Parpura V, Mothet JP and Zorec R Astrocytes as secretory cells of the central nervous system: Idiosyncrasies of vesicular secretion. EMBO J 35: 239-257, 2016.

28. John GR, Lee SC, Song X, Rivieccio M and Brosnan CF: IL-1-regulated responses in astrocytes: Relevance to injury and recovery. Glia 49: 161-176, 2005.

29. Niranjan R, Nath $C$ and Shukla R: The mechanism of action of MPTP-induced neuroinflammation and its modulation by melatonin in rat astrocytoma cells, C6. Free Radic Res 44: 1304-1316, 2010.

30. Adzic M, Stevanovic I, Josipovic N, Laketa D, Lavrnja I, Bjelobaba IM, Bozic I, Jovanovic M, Milosevic $M$ and Nedeljkovic N: Extracellular ATP induces graded reactive response of astrocytes and strengthens their antioxidative defense in vitro. J Neurosci Res 95: 1053-1066, 2017.

31. Pekny M, Levéen P, Pekna M, Eliasson C, Berthold $\mathrm{CH}$, Westermark B and Betsholtz C: Mice lacking glial fibrillary acidic protein display astrocytes devoid of intermediate filaments but develop and reproduce normally. EMBO J 14: 1590-1598, 1995.

32. Kraft AW, Hu X, Yoon H, Yan P, Xiao Q, Wang Y, Gil SC, Brown J, Wilhelmsson U, Restivo JL, et al: Attenuating astrocyte activation accelerates plaque pathogenesis in APP/PS1 mice. FASEB J 27: 187-198, 2013.

33. Calabrese F, Rossetti AC, Racagni G, Gass P, Riva MA and Molteni R: Brain-derived neurotrophic factor: A bridge between inflammation and neuroplasticity. Front Cell Neurosci 8: 430, 2014.

34. Huang S, Tong H, Lei M, Zhou M, Guo W, Li G, Tang X, Li Z, Mo M, Zhang X, et al: Astrocytic glutamatergic transporters are involved in A $\beta$-induced synaptic dysfunction. Brain Res 1678: 129-137, 2018.
35. Plá V, Paco S, Ghezali G, Ciria V, Pozas E, Ferrer I and Aguado F: Secretory sorting receptors carboxypeptidase E and secretogranin III in amyloid $\beta$-associated neural degeneration in Alzheimer's disease. Brain Pathol 23: 274-284, 2013.

36. Potokar M, Stenovec M, Kreft M, Kreft ME and Zorec R: Stimulation inhibits the mobility of recycling peptidergic vesicles in astrocytes. Glia 56: 135-144, 2008.

37. Fujita Y, Katagi J, Tabuchi A, Tsuchiya T and Tsuda M: Coactivation of secretogranin-II and BDNF genes mediated by calcium signals in mouse cerebellar granule cells. Brain Res Mol Brain Res 63: 316-324, 1999.

38. Kuczewski N, Porcher C, Ferrand N, Fiorentino H, Pellegrino C, Kolarow R,Lessmann V,Medina I and GaiarsaJL: Backpropagating action potentials trigger dendritic release of BDNF during spontaneous network activity. J Neurosci 28: 7013-7023, 2008.

39. Lou H, Kim SK, Zaitsev E, Snell CR, Lu B and Loh YP: Sorting and activity-dependent secretion of BDNF require interaction of a specific motif with the sorting receptor carboxypeptidase E. Neuron 45: 245-255, 2005.

40. Cawley NX, Rathod T, Young S, Lou H, Birch N and Loh YP: Carboxypeptidase E and Secretogranin III coordinately facilitate efficient sorting of proopiomelanocortin to the regulated secretory pathway in AtT20 cells. Mol Endocrinol 30: 37-47, 2016.

41. Moller JC, Kruttgen A, Burmester R, Weis J, Oertel WH and Shooter EM: Release of interleukin-6 via the regulated secretory pathway in PC12 cells. Neurosci Lett 400: 75-79, 2006. 